\title{
THE MISSING LINK: CULTURE AND LANGUAGE BARRIERS TO INTEROPERABILITY
}

\author{
Lawrence E. Whitman ${ }^{1}$, Hervé Panetto ${ }^{2}$, and Deemathie Desilva ${ }^{1}$ \\ ${ }^{1}$ Industrial \& Manufacturing Engineering Department, Wichita State University, USA \\ \{larry.whitman,deema.desilva\}@wichita.edu) \\ ${ }^{2}$ Research Centre for Automatic Control, University Henri Poincaré Nancy I, France \\ Herve.Panetto@cran.uhp-nancy.fr
}

\begin{abstract}
Interoperability is key to ensuring that a global supply chain operates as seamlessly as a vertically integrated organization. Much research has been accomplished and is on-going related to the technical, organisational and scientific issues concerning interoperating dissimilar enterprise systems and languages. However, there are significant issues concerning interoperating information across the barriers of cultures and national languages. This paper presents the key drawbacks regarding the cultural and language barriers to true exchange of knowledge. The paper then presents an example enterprise model in light of these identified issues. Copyright $@ 2006$ IFAC
\end{abstract}

Keywords: Enterprise integration, Enterprise modelling, Socio-technical issues.

\section{INTRODUCTION}

The activities within an enterprise are complex as companies manufacture a variety of products using different production methods to satisfy different customers. In order to face global competition and fluctuating market conditions, companies require the management of change (Vernadat, 1996). To simplify managing the change, Enterprise Integration (EI) plays an important role. Enterprise Integration consists of connecting and making interoperable all functional areas of an organization. Interoperability will improve organization's synergy in achieving its mission and vision in effective and efficient manner (Molina, et al., 2004). The process of integrating an enterprise is simplified by using a model. Enterprise models are often used to depict these various activities within an enterprise. An enterprise model is defined as "the art of externalising enterprise knowledge, which adds value to the enterprise or needs to be shared" (Vernadat, 2000). Developing an enterprise model provides a common understanding of the process and the associated activities.

There are various factors that drive changes to the internal processes of the organization. There are changes in market conditions due to competitor or customer related issues. The emergence of new technologies and changes due to change in product features exacerbates this speed of change (Harding \&
Popplewell, 1999). Basically, as the organization or business objective changes so must the enterprise model change to maintain the accuracy of information. Nowadays, the enterprise model is not reused to accommodate changes in the process. One reason is due to the inability of the enterprise to be aware of existing models.

An enterprise modelling language is required to create an enterprise model. There are many enterprise modelling languages and tools available and each modelling language has different characteristics (Petit, et al., 2002). "This intensive production of tools has led to a Tower of Babel situation in which the many tools, while offering powerful and distinct functionalities, are unable to interoperate and can hardly or not at all communicate and exchange models” (Panetto, et al., 2004b). Each enterprise modelling language and tool has its own characteristics, features and suitability for each type of industry and kind of modelling objective. It is impossible to force industries to only utilize one type of enterprise modelling language and one enterprise modelling tool. Diversity of enterprise models may create obstacles in achieving the goal of enterprise integration. To respond to these obstacles, interoperability of enterprise modelling languages is needed. 
IEEE (1990) defines interoperability as "the ability of two or more systems or components to exchange information and to use the information that has been exchanged.”.. Even though there are many different enterprise modelling languages, it would be productive if they can interchange with each other. In addition, interoperability leads to a standard for different languages. One example of interoperability language currently in development is called the Unified Enterprise Modelling Language (UEML) (Berio, et al., 2002; Panetto, et al., 2004a). UEML is not another enterprise modelling language, but it is a meta-model that supports interoperability of common enterprise modelling languages. The issue of interoperability has been dealt with extensively from a technical perspective, but only to a limited extent from a cultural and language perspective. This paper continues by discussing interoperability, continues with knowledge management, and then presents some issues with the exchange of knowledge.

\section{INTEROPERABILITY}

The word interoperability has many wide uses. The term interoperability is increasingly used in enterprise engineering and its related standardization activities (Chen \& Vernadat, 2002). To achieve interoperability, the systems need to interoperate their data, resources and business processes with semantics defined in a business context regardless of different languages, data formats, interfaces, executions platforms, communication protocols or message formats (Tsagkani, 2005). Interoperability is not only about transferring information but also performing an operation on behalf of another system.

One of the main obstacles to interoperability arises from the fact that the systems that support the functions in many enterprises were created independently, and do not share the same semantics for the terminology of their process models. Interoperability requires data stored in software systems on one machine to be sent and interpreted by another software system on another machine and for different purposes. To make these happen, standards on message format and transfer are needed.

\subsection{Levels of interoperability.}

To achieve interoperability of an enterprise, four levels are needed to achieve. The first level is technical interoperability, which then continues with syntactic, semantic and pragmatic interoperability.

Technical interoperability means transporting messages from one application to another. With today's technologies, technical interoperability is not the issue anymore. Current applications have full support in technical interoperability. Many applications have been able to understand each other. For example, a file from one application can be translated and read with a different application.
Syntactic interoperability is the second level. Before performing data exchange, the systems must agree on the format for data exchange. The Extensible Markup Language (XML) has solved this issue. XML has addressed the syntactic interoperability issue (Lilleng, 2005).

Semantic interoperability means understanding the content of messages and models in the same way by senders and receivers. Semantic interoperability refers to a system's ability for exchanging information inside organizations with heterogeneous information and-or between organizations without having to do tailoring to make this possible (Lilleng, 2005). Semantic interoperability is the focus of this paper.

Finally, pragmatic interoperability captures the willingness of partners for the actions necessary for the collaboration. This willingness to participate involves both capability of performing a requested action, and policies dictating whether the potential action is preferable for the enterprise to be involved in collaboration (Tsagkani, 2005).

Additionally, from a cultural point of view, organisational interoperability is a significant issue. If you compare occidental engineers and oriental engineers, you may think that the organisation of the work is influencing the interoperability

\section{MANAGING KNOWLEDGE IN ORGANIZATIONS}

This section presents the basic concepts involved in knowledge management. There are various indispensable components required for strategically managing knowledge. As an initial step, the company must first identify its knowledge assets and then it should share that knowledge across the knowledge network and learn from experience. There are three main aspects of knowledge management: storage, transfer and transformation of knowledge, which refer to the three main concepts of the General System Theory (Bertalanffy, 1969).

In this context, it is also important to discuss the various areas in which knowledge is dispersed in an organization:

Individual: Knowledge can be found in the hands of an individual worker who serves as a fundamental unit in the process of knowledge creation, storage, and use within the enterprise. Many times this knowledge is tacit and therefore not well documented.

Group: Group knowledge is more powerful than the sum of the knowledge acquired by an individual. This knowledge can be both formal and informal and is frequently intangible but is one of the most important knowledge assets within a company. 
Organizational: The organization, in turn, serves as a storehouse of knowledge with its own peculiar structure and divisions of functions, with multiple processes and activities to aid in the search for knowledge.

Knowledge links: Any organization has certain links with suppliers and customers and they exchange knowledge during their course of operation. This is believed to be more effective as inter-organizational links can provide more information than an isolated organization.

Finally, the common essence of models is to recognize the different levels of knowledge assets that reside within an organization. This will give an idea of where to look for information when you start the process of storing the knowledge with the help of models. Knowledge is inherently dynamic and may guarantee long term competitiveness. Therefore, it is important for every organization to be able to learn and update the knowledge base periodically. This will ensure model is constantly reused to maintain relevancy (Dutta, 1997).

\section{CULTURAL INTEROPERABILITY?}

As previously mentioned, semantic interoperability and knowledge interchange is the ultimate goal of an interoperability effort. The common understanding of the models leading to a better understanding of the processes leads to the final goal of pragmatic interoperability where the senders and receivers have the same actions in the same process. For this common action and understanding, cultural issues must be identified and addressed. This section lists some of those issues and provides examples.

When engineers design there is obviously much skill involved. However, there is also much tacit knowledge involved as well. An experienced engineer uses some tacit rules involved in their design process. There are many assumptions involved that are not explicitly stated. Design rules do not apply in every circumstance therefore they are not listed as design laws, but rather as design heuristics. In the US this is frequently referred to as "rules of thumb." Interestingly, there are similar terms in other languages. Koen (2003), presents several of these idioms:

- $\quad$ "In France le pif (the nose)

- In Germany, Faustregel (the fist)

- In Japan, (measuring with the eye)

- In Russia, (by the fingers)”

So, it is important to communicate this tacit knowledge as well. Unfortunately, tacit knowledge in one culture may be explicit and that which is explicit in one culture may be tacit in another. Frequently, enterprise models are considered to be 'documenting the obvious.' But, again what may be 'obvious' in one culture (and frequently between departments in a single enterprise) is not 'obvious' to others. Some research work is currently on-going in the domain of semantics annotation of models, based on common ontology, in order to deal with this issue (Boudjlida, et al., 2005) however real applicable solutions are fare away.

Language is not the only cultural issue in semantic interoperation. There is also the concern of different cultures having different design philosophies. Cultures have different constraints and different objectives. If engineering is, "design under constraint." Then, as those constraints (and objectives) change, so does the design process. Again, another example from Keon (2003, pg 76) concerning nuclear engineers in America and Russia. The American engineers tested many different designs before selecting the final design. Whereas, Russian engineers decided quickly on the design and then made it work. If the initial design choice is near optimum, the Russian method is better compared to the initial objective. If the initial design choice is not good, the American method is better as it allows alternate designs. So, in the exchange of a process model, each method carries with it an inherent design philosophy. If engineers from the two countries were collaborating on a joint design, a process model would help point out the difference in design (cultural) philosophies. However, some of these concepts are tacit in their nature and might not be explicit.

Culture impacts business. Also linguistic issues impact business in the context of culture. Successfully interchanging business and engineering information requires Intercultural Communication Power (ICP) (D. deSilva ref here). Whereas, engineers do not need to be completely culturally literate, the exchange of knowledge across dissimilar cultures in different native languages is imperative (Clark \& Jones, 1999).

The history of engineering progression is rich. The 1970s saw the incorporation of the factory with automation to allow the communication between different departments within the factory (CIM). The 1980s say the integration between engineering and manufacturing (CAD/CAM). The 1990s saw the integration of multiple sites and the beginning of supply chain integration. The $21^{\text {st }}$ century will saw the integration of multiple companies in what is called extended and virtual enterprises. With globalization all this integration must now take place across multiple countries with different languages and cultures.

\section{APPLICATION}

With the context of the previous sections, an example application of these concepts is presented. The example chosen is that of the engineering change process. The engineering change process is common 
to all designs. As engineers want to make the world a better place, they frequently do this by changing and improving existing designs. Designs are usually currently in the manufacturing process in different stages of completion. So, the engineering change process must communicate those changes to be incorporated into the manufacturing process. The process of how these changes are communicated and incorporated is the examples application for this paper.

When a need for a change is identified, An example of a method for the technical details can be found in Molina and Wright (2005). In this paper, the authors address the need for addressing more than the technical details, "Finally it is important to form, train and cultivate 'communities of e-engineering practice'. These communities must be formed with the mission to exchange experience, support collaborative learning, foster professional development and create the awareness to engage in trans-national and transcultural engineering partnerships” (Molina and Wright, 2005).

As engineers incorporate changes between companies, the incorporation of changes and the communication of knowledge is now required across the globe. The desire is not simply to exchange models, but rather to interoperate knowledge. In the context of the example, the process of incorporating design change intent must be communicated between all partners.

An example of a change order process is shown in figure 1 . The intent is to provide the basic complexity of the process. This process model was developed with an electronics manufacturing facility in the Midwest with facilities also located in the Ireland. A detail section of the process model is shown in figure 2. Figure 3 shows the same detail of the process model in another modelling language and in another language (French). The remainder of the paper will focus on the details of the process model shown in figure 2. Figure 2 shows that at a certain step in the process two asynchronous processes begin. The ECO and the associated BOM are analysed and this information is entered into the ORACLE database system. Two of these process steps include acronyms. There are three items that may appear as acronyms; ECO, BOMS, and ORACLE. Each of these terms is shown in both languages in table 1 . The term for bill of material is an acronym in English, but not in French. The term for the engineering change order is an acronym in both languages. The term Oracle may appear to be a strange word to anyone not familiar with the largest enterprise software vendor in the world may think that Oracle is an acronym or a strange term for engineers. To students just starting out, or even to a new engineer on the job, even this term may be confusing. To ensure commonality of terms, an ontology may be developed.
Ontology, as defined in philosophy, studies existence or being. In engineering and computer science, an ontology is defined as the conceptualization of a domain (Gruber, 1993). Therefore, an ontology can aid in defining the terms and understanding the context of the terms. However, the ontology must move beyond just the required technical terms, but should include the cultural and linguistic issues that can confuse the transfer of knowledge.

$\underline{\text { Table 1. Process Model Terms }}$

\begin{tabular}{ll}
\hline English & French \\
\hline BOM - Bill Of Material & Nomenclature \\
$\begin{array}{l}\text { ECO - Engineering } \\
\text { Change Order }\end{array}$ & $\begin{array}{l}\text { DMT - Demande de } \\
\text { modification technique }\end{array}$ \\
Oracle & Oracle
\end{tabular}

\section{CONCLUSIONS}

The main focus of this paper was to describe the nontechnical issues in interoperating enterprise models. This paper reviewed interoperability, the types of interoperability, and managing knowledge in organizations. Then issues concerning cultural interoperability were addressed. Finally, these concepts were presented in the context of an example in the engineering change process. The syntax, language, behaviour, plus cultural issues all inhibit the interoperation of knowledge leading to true semantic interoperability. Research and developments are still to be done to put these concepts into practice, either in enterprise modelling tools, either in the mind of all engineers. 

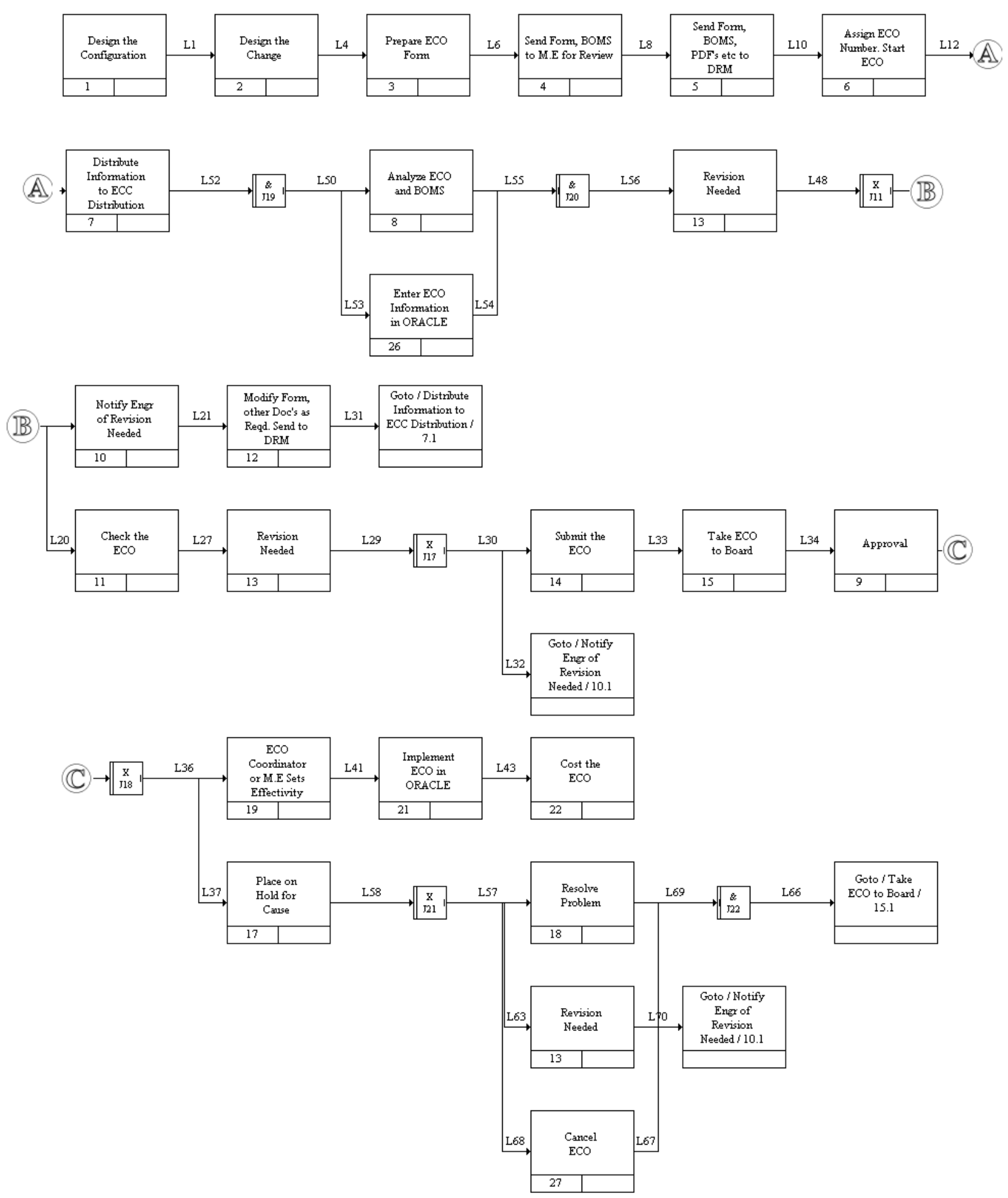

Figure 1. Process Model of Engineering Change Process 


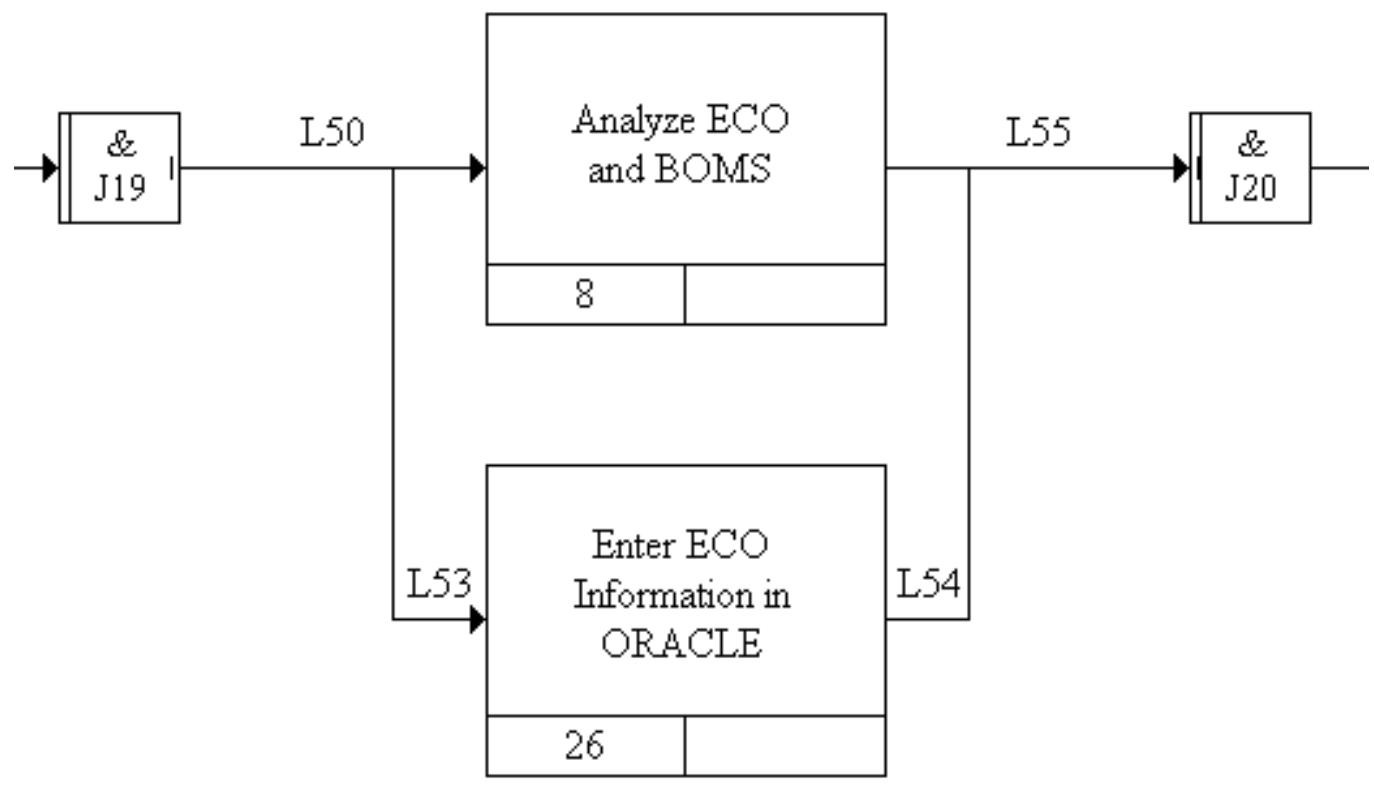

Figure 2. Section of Process Model

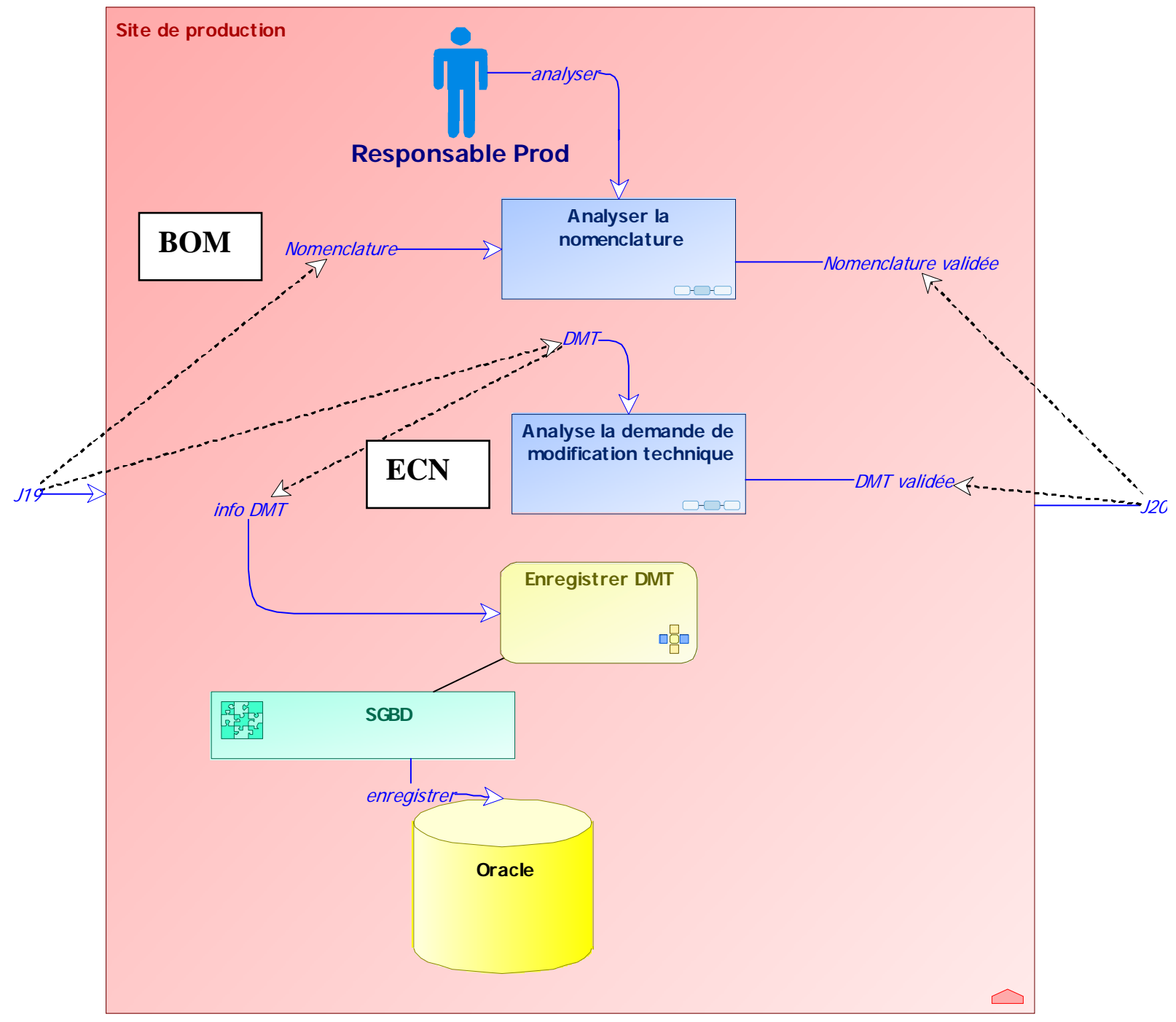

Figure 3. Section of Process Model in another Tool and another language 


\section{REFERENCES}

Berio G., et al. (2002). Requirements analysis: initial core constructs and architecture, UEML TN IST 2001 34229. http://www.ueml.org, 2002.

Bertalanffy L. V. (1969). General System Theory: foundations, development, applications. George Braziller, New York.

Boudjlida N., Dong C., Baina S., Panetto H., Haussmann K., Tomas J., Abian M., Nunez M.J. (2005). Deliverable DTG4.1: A practical experiment on semantic enrichment in a homogeneous environment. In Interoperability Research for Networked Enterprises Applications and Software Network of Excellence, $n^{\circ}$ IST 508011, November 2005

Chen, D., and Vernadat, F.B. (2002). Enterprise Interoperability: A Standardization View. In Proceedings of the IFIP International Conference on Enterprise Integration and Modelling Technology (ICEIMT'02), Kluwer Academics Publisher, Valencia, Spain, April 24-26 2002.

Clark, T. and R. Jones (1999). Organisational Interoperability Maturity Model for C2, In Proceedings of the Command And Control Research And Technology Symposium (CCRTS), June 29 - July 1, Newport, RI, USA

deSilva, D. and deSilva, D. (1995). How to gain a competitive edge in international business through inter-cultural communication power. In: Proceedings of Pan-Pacific Conference XII: A Business, Economic, and Technological Exchange. May 29-June 1, 1999. Dunedin, New Zealand.

Dutta, S., T. Gulledge, and M. Tarimcilar (1997), Strategies for implementing knowledge based systems. IEEE transaction on Engineering Management, 44(1), 79-91.

Gruber T. R. A (1993). Translation approach to portable ontologies. Knowledge Acquisition, 5(2), 199-220.

Harding, J., B. Yu, and K. Popplewell (1999), Information modeling: an integration of views of a manufacturing enterprise. International Journal of Production Research, 37, 12, 2777-2792.

IEEE (1990). IEEE Standard Computer Dictionary: A Compilation of IEEE Standard Computer Glossaries. Institute of Electrical and Electronics Engineers. New York, NY: 1990.

Koen, B. (2003). Discussion of the Method: Conducting the Engineer's Approach to Problem Solving. Oxford University Press. USA.

Lilleng, J. (2005). Towards Semantic Interoperability. Proceedings of the IFIP/ACM SIGAPP INTEROP-ESA conference, February 23-25, Geneva, Switzerland, Springer Science publisher.

Molina, A., Chen, D., Panetto, H., Vernadat, F., \& Whitman, L. (2004). Enterprise integration and networking: issues, trends and vision. In Proceedings of the IFIP International Conference on Enterprise Integration and
Modeling Technology (ICEIMT'04), Knowledge Sharing in the Integrated Enterprise Interoperability - Strategies for the Enterprise Architect, 183, Springer publisher, October 9th11th, Toronto, Canada.

Molina, A., Aca, J., and Wright, P. (2005). Global collaborative engineering environment for integrated product development. In International Journal of Computer Integrated Manufacturing 18( 8), pp. 635-651.

Panetto H., G. Berio, K. Benali, N. Boudjlida and M. Petit (2004a). A Unified Enterprise Modelling Language for enhanced interoperability of Enterprise Models. In Proceedings of IFAC INCOM 2004 Symposium, April 5th-7th, Bahia, Brazil.

Panetto, H., Whitman, L.E. and Chatha, K.A. (2004b). Ontology for Enterprise Interoperability in the domain of Enterprise Business Modelling. In: IFAC TC 5.3 International workshop on Enterprise Interoperability, Integration and Networking (EI2N'2005), Proceedings of the Worlshops of the IFIP/ACM SIGAPP INTEROPESA conference, February 22nd, Geneva, Switzerland, pp. 103-114, Hermès Science publishing, ISBN 1-903209-49-5

Petit M, and al. (2002). State of the Art in Enterprise Modelling, UEML TN IST 2001 34229, www.ueml.org

Tsagkani, C. (2005) Inter-Organizational Collaboration on the Process Layer. In Proceedings of the IFIP/ACM SIGAPP INTEROP-ESA conference, February 23-25, Geneva, Switzerland, Springer Science publisher, ISBN: 1-84628-151-2

Vernadat, F.B. (1996). Enterprise Modeling and Integration: Principles and Applications. London: Chapman \& Hall.

Vernadat, F.B. (2000). UEML: towards a unified enterprise modeling language. International Journal Production Research, 40(17), pp. 43094321. 